\title{
Identification of microRNAs and mRNAs associated with multidrug resistance of human laryngeal cancer Hep-2 cells
}

\author{
Wanzhong Yin ${ }^{1}$, Ping Wang ${ }^{1}$, Xin Wang ${ }^{1}$, Wenzhi Song ${ }^{2}$, Xiangyan Cui ${ }^{1}$, Hong Yu ${ }^{1}$ and Wei Zhu ${ }^{1}$ \\ ${ }^{1}$ Department of Otorhinolaryngology, Head and Neck Surgery, The First Clinical Hospital, Norman Bethune College of Medicine, \\ Jilin University, Changchun, China \\ ${ }^{2}$ Department of Stomatology, China-Japan Friendship Hospital, Jilin University, Changchun, China
}

\begin{abstract}
Multidrug resistance (MDR) poses a serious impediment to the success of chemotherapy for laryngeal cancer. To identify microRNAs and mRNAs associated with MDR of human laryngeal cancer Hep-2 cells, we developed a multidrug-resistant human laryngeal cancer subline, designated Hep-2/v, by exposing Hep-2 cells to stepwise increasing concentrations of vincristine $(0.02-0.96 \mu \mathrm{M})$. Microarray assays were performed to compare the microRNA and mRNA expression profiles of Hep-2 and Hep-2/v cells. Compared to Hep-2 cells, Hep-2/v cells were more resistant to chemotherapy drugs ( $\sim 45$-fold more resistant to vincristine, 5.1-fold more resistant to cisplatin, and 5.6-fold more resistant to 5-fluorouracil) and had a longer doubling time $(42.33 \pm 1.76$ vs $28.75 \pm 1.12 \mathrm{~h}, \mathrm{P}<0.05)$, higher percentage of cells in $\mathrm{G} 0 / \mathrm{G} 1$ phase $(80.98 \pm 0.52$ vs $69.14 \pm 0.89, \mathrm{P}<0.05)$, increased efflux of rhodamine $123(95.97 \pm 0.56$ vs $12.40 \pm 0.44 \%, \mathrm{P}<0.01)$, and up-regulated MDR1 expression. A total of 7 microRNAs and 605 mRNAs were differentially expressed between the two cell types. Of the differentially expressed mRNAs identified, regulator of G-protein signaling 10 , high-temperature requirement protein A1, and nuclear protein 1 were found to be the putative targets of the differentially expressed microRNAs identified. These findings may open a new avenue for clarifying the mechanisms responsible for MDR in laryngeal cancer.
\end{abstract}

Key words: Multidrug resistance; Laryngeal cancer; Hep-2 cells; mRNA; microRNA

\section{Introduction}

Laryngeal cancer (LC) is one of the most common head and neck cancers. Surgery-based multimodality therapy is currently the most effective treatment for LC. Chemotherapy, as an important therapeutic modality for LC, plays a crucial role in killing residual tumor cells, preventing tumor micrometastasis, and lowering the incidence of local recurrence or distant metastases (1). However, multidrug resistance (MDR) poses a serious impediment to the success of chemotherapy for LC (2).

The acquisition of MDR by tumor cells is a complicated, multifactorial process. Several interrelated mechanisms have been proposed for the explanation of the emergence of MDR $(3,4)$. These mechanisms include i) reduced drug accumulation due to increased expression of efflux drug transporters, such as MDR1 and MDRassociated proteins (5), ii) increased drug inactivation resulting from metabolic alterations (6), iii) increased ability to repair and/or tolerate DNA lesions due to increased expression of DNA topoisomerases (7), and iv) inhibition of apoptosis by alteration in the expression of apoptosis-associated genes or proteins, such as p53 and $\mathrm{Bcl}-2(8,9)$. Understanding the precise mechanisms by which tumor cells acquire MDR is imperative for the discovery of novel anti-MDR agents.

MicroRNAs (miRNAs) are a class of recently discovered small non-coding RNAs that play important regulatory roles in cell proliferation, differentiation, and apoptosis by targeting mRNAs for degradation or translational repression $(10,11)$. Deregulated miRNA expression has been linked to the development and progression of a variety of cancers, including LC (12-14). miRNAs have also been strongly implicated in the modulation of MDR in many different types of cancer, such as breast, gastric, ovarian, and prostate cancer (15-19). Multiple MDRassociated miRNA targets, such as Bcl-2 (17), PTEN (18), and MDR1 (20), have been identified. However,

Correspondence: Wei Zhu, Department of Otorhinolaryngology, Head and Neck Surgery, The First Clinical Hospital, Norman Bethune College of Medicine, Jilin University, 71 Xinmin Street, Changchun, 130000, China. Fax: +86-431-8878-6160. E-mail: zhuwei3000@yahoo.com.cn 
there have been no reports of miRNAs involved in MDR in LC.

To better understand the mechanism underlying MDR in LC and gain an insight into the role of miRNAs in MDR of LC cells, we developed a multidrug resistant variant (designated Hep-2/v) of the human LC line Hep-2 in the present study. Given that vincristine (VCR) is an important component of many combination chemotherapy regimens for treating LC and that MDR of VCR-resistant cell lines has been demonstrated in previous studies $(21,22)$, we selected VCR to induce MDR of Hep-2 cells. By comparing the expression profiles of miRNAs and mRNAs of Hep-2 cells and Hep-2/v cells using microarray assays, we identified multiple miRNAs and mRNAs that are potentially involved in MDR of LC cells. Furthermore, the association between these miRNAs and mRNAs was analyzed using an miRNA target prediction program. The results obtained provide new clues into the molecular mechanisms behind MDR in LC.

\section{Material and Methods}

\section{Cell culture and treatment}

The human LC cell line Hep-2 was provided by the Chinese Academy of Medical Sciences (Beijing, China). Cells were cultured in RPMI 1640 medium (Invitrogen, USA) supplemented with $10 \%$ fetal calf serum (FCS, Invitrogen), $100 \mathrm{U} / \mathrm{mL}$ penicillin, and $100 \mathrm{U} / \mathrm{mL}$ streptomycin at $37^{\circ} \mathrm{C}$ in a humidified atmosphere containing $5 \% \mathrm{CO}_{2}$. VCR (Sigma, USA) was added to Hep-2 cells in stepwise increasing concentrations (from 0.02 to $0.96 \mu \mathrm{M}$ ) to develop a drugresistant cell variant (Hep-2/v). The Hep-2/v cell line was obtained after a total of 40 passages in the presence of VCR. The MDR phenotype of Hep-2/v cells was determined by incubating the cells with serially diluted VCR $(8-128 \mu \mathrm{M})$, cisplatin (DDP, 0.625-10 $\mu \mathrm{M}$; Sigma), and 5-fluorouracil (5-FU, 62.5-1,000 $\mu \mathrm{M}$; Sigma).

\section{Morphological observations}

For light microscopy, Hep-2 or Hep-2/v cells in log phase growth were seeded into culture flasks, allowed to grow to $80 \%$ confluence, and observed under an inverted microscope (Olympus, Japan). For transmission electron microscopy, Hep-2 or Hep-2/v cells were harvested by digestion with $0.25 \%$ trypsin, fixed with $2.5 \%$ glutaraldehyde, post-fixed with $2 \%$ osmium tetroxide, and processed for section preparation as previously described (23). The samples were observed using a JEM 1200EX transmission electron microscope (JEOL, Japan).

\section{Cell growth studies}

Hep-2 or Hep-2/v cells in log phase growth were adjusted to a density of $5 \times 10^{4} / \mathrm{mL}$, seeded on 24-well plates, and cultured at $37^{\circ} \mathrm{C}$ in a humidified atmosphere containing $5 \% \mathrm{CO}_{2}$. The mean number of adherent cells in three wells was calculated each day for 7 days to plot growth curves. The doubling time of the cells in log phase growth was calculated using the following formula: doubling time $=\mathrm{T} \times \log 2 /(\log \mathrm{Nt}-\log \mathrm{No})$, where No and $\mathrm{Nt}$ represent the number of cells at the beginning and at the end of culture during time $\mathrm{T}$, respectively.

\section{MTT assay}

Hep-2 or Hep-2/v cells were digested with $0.25 \%$ trypsin to prepare single cell suspensions. After adjusting the cell density to $5 \times 10^{4}$ cells $/ \mathrm{mL}$, the cells were seeded at $100 \mu \mathrm{L} /$ well on 96-well plates in triplicate and exposed to serially diluted VCR $(8-128 \mu \mathrm{M})$, DDP $(10-160 \mu \mathrm{M})$, or 5 -FU $(1,000-16,000 \mu \mathrm{M})$ for $72 \mathrm{~h}$, followed by incubation with MTT solution for $4 \mathrm{~h}$. RPMI 1640 medium was used as a blank control. At the end of the incubation period, dimethyl sulfoxide was added at $200 \mu \mathrm{L} /$ well and the plates were incubated in an air bath shaker at $37^{\circ} \mathrm{C}$ for $5 \mathrm{~min}$. Absorbance at $490 \mathrm{~nm}\left(\mathrm{~A}_{490}\right)$ was measured using a microplate reader to assess cell viability. The doseresponse curve was then plotted to determine the halfmaximal-inhibitory concentration $\left(\mathrm{IC}_{50}\right)$. The initial concentration of VCR was equal to half of the $\mathrm{IC}_{50}$ value. The resistance index $(\mathrm{RI})$ was calculated by dividing the $\mathrm{IC}_{50}$ values obtained for Hep-2/v cells by those for Hep-2 cells.

\section{Rhodamine 123 retention assay and cell cycle analysis by flow cytometry}

After Hep-2 or Hep-2/v cells $\left(2 \times 10^{6}\right)$ were harvested to prepare single cell suspensions, $2.5 \mu \mathrm{L}$ rhodamine 123 ( $5 \mathrm{mM}$; Sigma) was added and incubated at $37^{\circ} \mathrm{C}$ for $30 \mathrm{~min}$. The cells were then centrifuged at $60 \mathrm{~g}$ to remove the supernatant, washed with fresh medium, and then incubated at $37^{\circ} \mathrm{C}$ for an additional $10 \mathrm{~min}$. After washing the cells again with fresh medium, the cells were resuspended in precooled medium and subjected to flow cytometric measurement of rhodamine 123 retention. For cell cycle analysis, Hep- 2 or Hep-2/v cells $\left(2 \times 10^{6}\right)$ were harvested and fixed with a mixture of $300 \mu \mathrm{L}$ phosphatebuffered saline (PBS) containing 5\% FCS and $700 \mu \mathrm{L}$ $70 \%$ ethanol at $-20^{\circ} \mathrm{C}$ for $24 \mathrm{~h}$. After washing twice with PBS, the cells were incubated with $100 \mu \mathrm{L}$ RNase A $(1 \mathrm{mg} / \mathrm{mL})$ at $37^{\circ} \mathrm{C}$ for $30 \mathrm{~min}$, stained with $300 \mu \mathrm{L}$ propidium iodide $(100 \mu \mathrm{g} / \mathrm{mL})$ at room temperature for $20 \mathrm{~min}$, and analyzed by flow cytometry. A BD FACSAria flow cytometer (BD Biosciences, USA) was used, and data were analyzed using the QuantiCALC software (BD Biosciences).

\section{miRNA microarray analysis}

Total RNA was prepared from Hep-2 or Hep-2/v cells using Trizol reagent (Invitrogen), purified using the mirVana miRNA Isolation kit (Ambion, USA), tailed with polyadenylation polymerase, ligated with biotinylated 3DNA dendrimers, and hybridized to Affymetrix GeneChip miRNA arrays using the FlashTag ${ }^{\mathrm{TM}}$ Biotin 
RNA Labeling kit (Genisphere, USA) according to the manufacturer's instructions. Slides were scanned with the Affymetrix GeneChip Scanner 3000 (Affymetrix, USA), and miRNA data were analyzed using the miRNA QC Tool (Affymetrix). Differentially expressed miRNA genes were identified using the significance analysis of microarrays (SAM) program. The miRNAs with a q-value $<5 \%$ and a fold-change $>2$ were considered to be significantly differentially expressed. Hierarchical clustering of differentially expressed miRNAs was performed using the Cluster 3.0 program.

\section{mRNA microarray analysis and miRNA target prediction}

Total RNA was prepared from Hep-2 or Hep-2/v cells as described above. RNA purification and cDNA synthesis as well as cRNA synthesis, purification, labeling, and hybridization to Human Genome v2.0 oligonucleotide microarrays were performed using the cRNA Amplification and Labeling Kit (CapitalBio, China) according to the manufacturer's instructions. Slides were scanned with the LuxScan $10 \mathrm{KA}$ microarray scanner (CapitalBio), and mRNA data were analyzed using the LuxScan3.0 image analysis software (CapitalBio). Differentially expressed mRNAs were detected using the SAM program. Genes with a q-value $<5 \%$ and a foldchange $>2$ were considered to be significantly differentially expressed. Hierarchical clustering of differentially expressed genes was performed using the Cluster 3.0 program. Based on the data obtained from differentially expressed miRNA genes and mRNAs, miRNA target prediction was performed. Putative target genes of each miRNA were predicted using the miRanda software. We then analyzed the mRNA and miRNA data to select the differentially expressed genes based on the direction of expression change, which was where the up-regulated miRNA was associated with a down-regulated, predicted mRNA target, or otherwise.

Quantitative real-time reverse transcriptase-polymerase chain reaction (RT-PCR) analysis of $\mathrm{MRNA}$ and miRNA expression

Total RNA (the same samples for microarray analysis) was reversely transcribed into cDNA using M-MLV (Moloney murine leukemia virus) reverse transcriptase (Invitrogen) according to the manufacturer's instructions. Real-time PCR was then performed to determine the expression levels of MDR1, regulator of G-protein signaling 10 (RGS10), high-temperature requirement protein A1 (HTRA1), nuclear protein 1 (NUPR1), hasmiR-210, has-miR-923, and has-miR-93. Beta-actin and U6 small nuclear RNA (snRNA) were used as controls for quantification. The primers used for MDR1, RGS10, HTRA1, NUPR1, beta-actin, glyceraldehyde-3-phosphate dehydrogenase (GAPDH), and U6 snRNA are as follows: MDR1 (304 bp): 5'-GCA CTA AAG TAG GAG ACA AAG
GAA-3', 5'-TGA CTC TGC CAT TCT GAA ACA C-3'; RGS10 (304 bp): 5'-GGC CGC CGT CAG ACA TCC AC3', 5'-AGC CGA GAC TGC CCC TCC AC-3'; HTRA1 (210 bp): 5'-TGC CTG TCC TGC TGC TTG GC-3', 5' ACG GGC CTC CCG AGT TTC CA-3'; NUPR1 (358 bp): 5'-GGC TGG ACT CAG GGA CCG ACT-3', 5' -TCC GGC CTC CAC CTC CGA-3'; beta-actin (250 bp): 5'-CAT GTA CGT TGC TAT CCA GGC-3', 5'-CTC CTT AAT GTC ACG CAC GAT-3'; GAPDH (306 bp): 5'-TGA ACG GGA AGC TCA CTG G-3', 5'-TCC ACC ACC CTG TTG CTG TA-3'; U6 snRNA (94 bp): 5'-CTC GCT TCG GCA GCA CA-3', 5'-AAC GCT TCA CGA ATT TGC GT-3'. For miRNA amplification, a universal sense primer (5'-GTG CAG GGT CCG AGG T-3') and an miRNA-specific antisense primer (has-miR-210: 5'-TGT GCG TGT GAC AGC GGC-3'; has-miR-923: 5'-GGT CAG CGG AGG AAA AGA A-3'; has-miR-93: 5'-CAA AGT GCT GTT CGT GCA GG-3') were used. The expression level of each mRNA or miRNA was measured using the $2^{-\Delta \Delta C t}$ method.

\section{Western blot}

Total cell extracts were prepared and subjected to spectrophotometric measurement of protein concentration. Forty micrograms of total cell proteins was separated by sodium dodecyl sulfate-polyacrylamide gel electrophoresis and transferred to a polyvinylidene difluoride membrane (Bio-Rad, USA). The membrane was blocked for $1 \mathrm{~h}$ at room temperature in PBS containing $0.3 \%$ Tween 20 and $5 \%$ skim milk and incubated overnight at $4^{\circ} \mathrm{C}$ with an anti-MDR1 antibody (dilution 1:1,000; Chemicon, USA) or anti-actin antibody (dilution 1:1,500; Chemicon). Antibody binding was revealed by incubation with horseradish peroxidase-coupled secondary antibody (dilution 1:5,000; Pierce, USA) for $1 \mathrm{~h}$ at room temperature. Chemiluminescence was detected using enhanced chemiluminescence reagents (Pierce). The relative level of the MDR1 protein to actin was determined by densitometric scanning.

\section{Statistical analysis}

Statistical analysis was performed using the SPSS 11.0 software package (SPSS Inc., USA). Data are reported as means $\pm S D$. The means between two groups were compared using the Student $t$-test. The comparison of multiple means was performed using analysis of variance. Categorical data were compared using the chi-square test.

\section{Results}

\section{Successful generation of a multidrug-resistant Hep-2 subline (Hep-2/v)}

A multidrug-resistant variant of the human LC line Hep-2 was selected by adaptation to stepwise increasing concentrations of VCR and designated as Hep-2/v. The $\mathrm{IC}_{50}$ values for VCR, DDP, and 5-FU were significantly 
higher in Hep-2/v cells than in Hep-2 cells (VCR $=0.04 \pm 0.01$ vs $1.8 \pm 0.20 ; \mathrm{DDP}=0.59 \pm 0.26$ vs $1.97 \pm 0.17 ; 5-\mathrm{FU}=60.92 \pm 4.23$ vs $330 \pm 4.65$; all $\mathrm{P}<0.05)$, and the $\mathrm{RI}$ for $\mathrm{VCR}$, DDP, and 5-FU were $45.00,5.10$, and 5.56, respectively.

\section{Multidrug-resistant Hep-2/v cells exhibited altered} biological characteristics compared to Hep-2 cells

Hep-2/v cells showed significant morphological changes compared to Hep-2 cells. Both Hep-2 and Hep$2 / v$ cells grew in an adherent manner. Hep-2/v cells were larger and rounder, had more cytoplasmic granules, and aggregated into small clusters. The adherence ability of Hep-2/v cells was weaker than that of Hep-2 cells. Electron microscopy showed projected microvilli on the cell surface and abundant organelles in Hep-2 cells (see Supplementary Figure S1). In contrast, loss of surface microvilli, slight nuclear vacuolation, organelle swelling, and a marked reduction in the number of organelles were observed in Hep-2/v cells.

Hep-2/v cells grew slower than Hep-2 cells. Figure 1A shows the growth curves of Hep-2 and Hep-2/v cells. The doubling time of Hep-2/v cells was significantly longer than that of Hep-2 cells $(42.33 \pm 1.76$ vs $28.75 \pm 1.12 \mathrm{~h}$, $\mathrm{P}<0.05$ ), indicating that the growth rate of multidrugresistant Hep-2/v cells was slower than that of wild-type Hep-2 cells.

Hep-2/v cells in G0/G1 phase arrest compared to Hep-2 cells. Flow cytometric analysis showed that the percentage of cells in G0/G1 phase was significantly higher in Hep-2/v cells than in Hep-2 cells $(80.98 \pm 0.52$ vs $69.14 \pm 0.89$, $\mathrm{P}<0.05)$, whereas the percentage of cells in $\mathrm{S}$ phase was significantly lower in Hep-2/v cells than in Hep-2 cells $(9.76 \pm 0.88$ vs $19.43 \pm 2.04, \mathrm{P}<0.05)$. No significant difference was noted in the percentage of cells in G2/M phase in the two groups of cells $(8.99 \pm 0.48$ vs $11.52 \pm 1.20$, respectively, $\mathrm{P}>0.05$; Figure $1 \mathrm{~B}$ ).

Hep-2/v cells had increased efflux of rhodamine 123 compared to Hep-2 cells. As shown in Figure 2A, the percentage of rhodamine 123-positive Hep-2 cells was significantly higher than that of rhodamine 123-positive Hep-2/v cells $(95.97 \pm 0.56$ vs $12.40 \pm 0.44 \%$, respectively, $\mathrm{P}<0.01$ ), suggesting an increased efflux of rhodamine 123 in multidrug-resistant Hep-2/v cells.

MDR1 mRNA and protein expression is upregulated in Hep-2/v cells compared to Hep-2 cells. As shown in Figure 2B and $\mathrm{C}$ and Supplementary Figure $\mathrm{S} 2$, the relative expression levels of MDR1 MRNA and protein were 9.61- and 3.48-fold higher in Hep-2/v cells than in Hep-2 cells (both $\mathrm{P}<0.01$ ).

\section{Identification of 7 putative MDR-associated miRNAs in Hep- $2 / v$ cells}

A total of 7 differentially expressed miRNAs were identified when Hep-2 and Hep-2/v cells were compared by microarray analysis (Table 1 and Figure $3 \mathrm{~A}$ ). Two
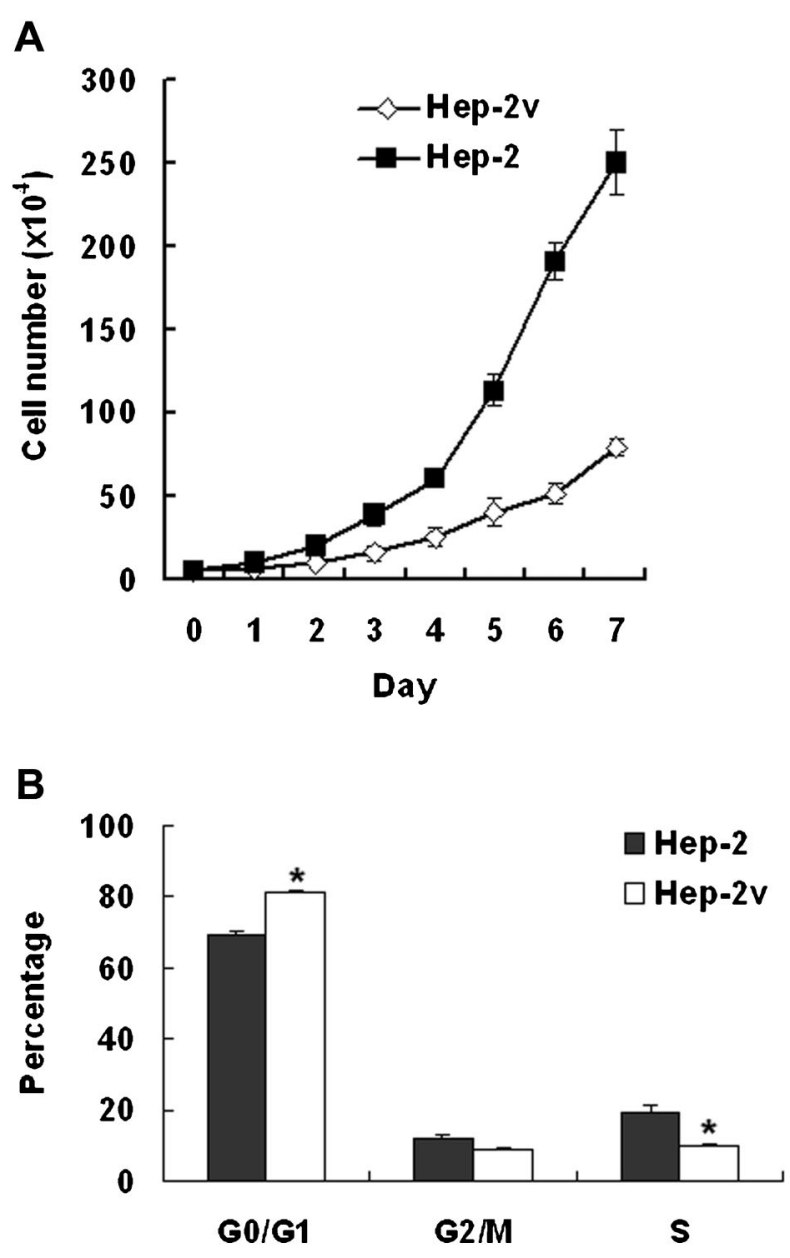

Figure 1. Hep-2/v cells grow slower and have a higher percentage arrest in G0/G1 phase compared to Hep-2 cells. A, Growth curves of Hep-2 and Hep-2/v cells. The data shown represent average values for triplicate cultures and are reported as means $\pm S D$. $B$, Cell cycle distribution of Hep-2 and Hep-2/v cells. Hep-2 or Hep-2/v cells were stained with propidium iodide and analyzed by flow cytometry. Data are reported as mean $\pm S D$ percentages of cells in different phases. ${ }^{*} \mathrm{P}<0.05$ vs Hep-2 cells (chi-square test).

miRNAs (has-miR-210 and has-miR-923) were significantly up-regulated and five miRNAs (has-miR-93, hasmiR-93-star, has-miR-424-star, has-miR-25-star, and has-miR-494) were significantly down-regulated in Hep$2 / v$ cells. To verify the reliability of the microarray results, real-time RT-PCR was performed to measure the expression of has-miR-210, has-miR-923, and has-miR93. As shown in Figure 4A, RT-PCR results showed that has-miR-210 and has-miR-923 expression had a 4.78and 1.53-fold up-regulation, and has-miR-93 expression showed a 1.46-fold down-regulation in Hep-2/v cells compared with Hep-2 cells. These findings were well matched with the microarray results. 


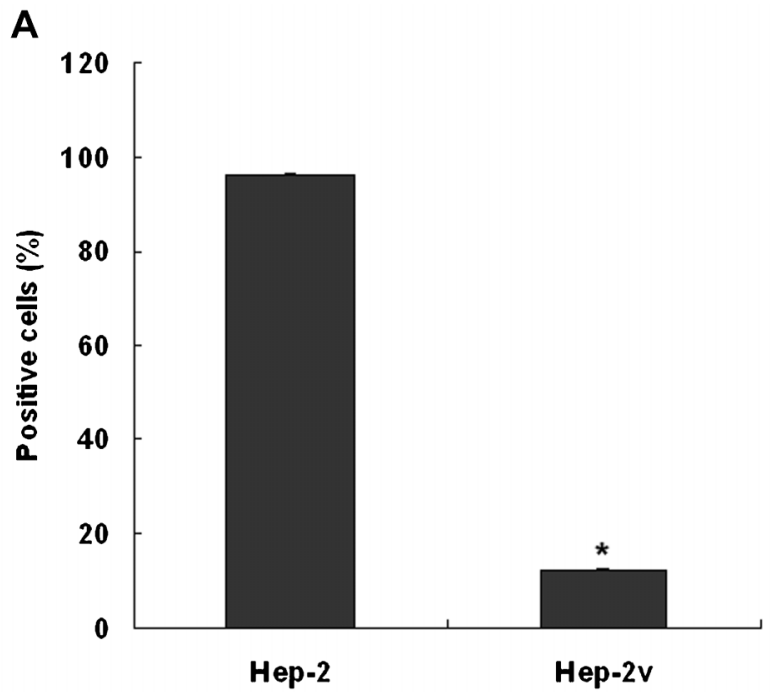

B

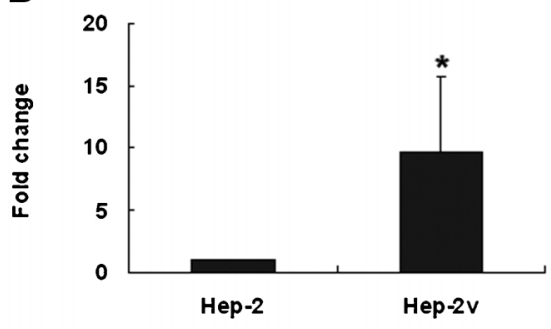

C

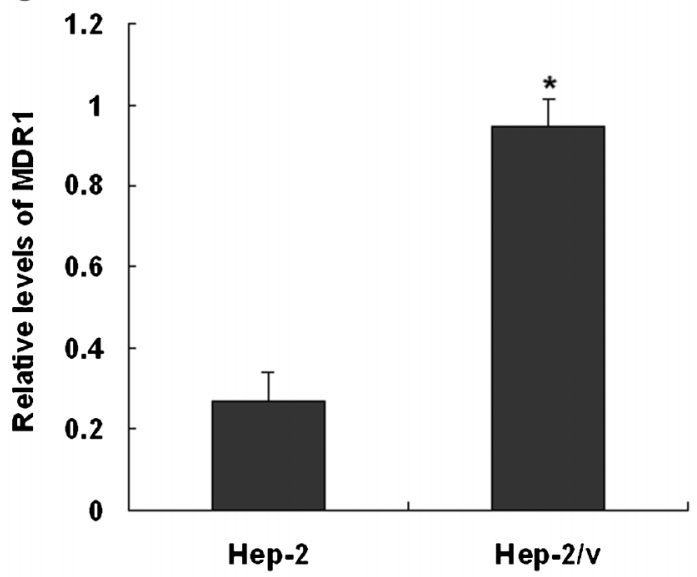

Identification of 605 putative MDR-associated mRNAs in Hep-2/v cells

A total of 605 differentially expressed mRNAs were identified when Hep-2 and Hep-2/v cells were compared by microarray analysis (Figure 3B, Supplementary Figure S3, and Supplementary Table S1). Of these mRNAs, 270 were significantly up-regulated and 335 were significantly down-regulated in Hep-2/v cells. These mRNAs have
Figure 2. Rhodamine 123 efflux is increased and multidrug resistance 1 (MDR1) expression is up-regulated in Hep-2/v cells compared to Hep-2 cells. A, Rhodamine 123 retention in Hep-2 and Hep-2/v cells. The cells were incubated with rhodamine 123 and measured by flow cytometry. Data are reported as mean \pm SD percentages of rhodamine 123-positive cells. ${ }^{*} \mathrm{P}<0.01$ vs Hep-2 cells (chi-square test). $B$, Expression of MDR1 mRNA in Hep-2 and Hep-2/v cells. The relative levels of MDR1 mRNA in Hep-2 and Hep-2/v cells were determined by real-time reverse transcription-polymerase chain reaction. Data are reported as means \pm SD. ${ }^{*} \mathrm{P}<0.01$ vs Hep-2 cells (Student $t$ test). $C$, Expression of the MDR1 protein in Hep-2 and Hep-2/v cells. The relative levels of the MDR1 protein in Hep-2 and Hep-2/ $\checkmark$ cells were determined by Western blotting. Data are reported as means $\pm \mathrm{SD}$. ${ }^{*} \mathrm{P}<0.01$ vs Hep-2 cells (Student $t$-test).

been implicated in carcinogenesis, signal transduction, cytoskeletal organization and cell motility, protein translation, DNA synthesis, and repair and metabolism, as well as other cellular functions. Table 2 shows representative mRNAs that were differentially expressed between Hep-2 and Hep-2/v cells. Criteria for choosing these representative mRNAs were: i) showing significantly differential expression, ii) having either a direct or indirect relationship with the identified differentially expressed miRNAs (revealed by searching miRNA target predication databases), and iii) genes of our interest for further study.

HTRA1 and NUPR1 expression was down-regulated and RGS10 expression was up-regulated in Hep-2/v cells compared to Hep-2 cells

To identify putative target genes of differentially expressed miRNAs, we used the miRanda software to deduce target genes. NUPR1 was identified as the putative target of has-miR-210 and has-miR-923, whereas HTRA1 and RGS10 were identified as putative targets of has-miR-210 and has-miR-93, respectively. Real-time RT-PCR analysis confirmed that the relative expression levels of HTRA1 and NUPR1 mRNA were significantly lower (HTRA1: 2.12- and 5.08-fold relative to beta-actin and GAPDH, respectively; NUPR1: 2.81- and 4.06-fold relative to beta-actin and GAPDH, respectively), whereas the RGS10 mRNA expression level was

Table 1. Differential expression of miRNAs by Hep-2 and Hep-2/v cells.

\begin{tabular}{lccc}
\hline miRNA & Fold-change & Regulation & q-value \\
\hline has-miR-210 & 4.69 & up & 4.06 \\
has-miR-923 & 2.30 & up & 4.06 \\
has-miR-93 & 0.44 & down & 0.00 \\
has-miR-93-star & 0.44 & down & 0.00 \\
has-miR-424-star & 0.45 & down & 0.00 \\
has-miR-25-star & 0.42 & down & 0.00 \\
has-miR-494 & 0.31 & down & 0.00 \\
\hline
\end{tabular}

$\operatorname{miRNA}=$ microRNA. 



Figure 3. Hierarchical clustering of differentially expressed microRNAs $(A)$ and mRNAs $(B)$ between Hep-2 and Hep-2/v cells.

significantly higher (2.35- and 2.71-fold relative to betaactin and GAPDH, respectively) in Hep-2/v cells (Figure 4B).

\section{Discussion}

MDR is a major obstacle to the successful chemotherapeutic treatment of LC, and therefore the elucidation of the mechanisms responsible for MDR in LC represents an important step toward overcoming this problem. The establishment of multidrug-resistant tumor cell lines provides an important tool for the study of MDR in cancer (24). In the present study, we generated a multidrugresistant human LC subline, which was designated Hep-2/ $\mathrm{v}$, by exposing Hep-2 cells to stepwise increasing concentrations of VCR. In comparison to Hep-2 cells, Hep-2/v cells showed approximately 45 -fold resistance to VCR, 5.1-fold resistance to DDP, and 5.6-fold resistance to $5-\mathrm{FU}$. Thus, the Hep-2/v subline may represent a promising cell model for the study of MDR and screening of anti-MDR agents in LC.

The doubling time of Hep-2/v cells was $13.58 \mathrm{~h}$ longer than that of Hep-2 cells, suggesting that the growth rate of Hep-2/v cells decreased remarkably. Because chemotherapy mainly affects rapidly dividing cells, tumor cells with a long doubling time are generally insensitive to chemotherapy (25). The slower growth rate of Hep-2/v cells could be explained by the observation that the percentage of cells in G0/G1 phase significantly increased and the percentage of cells in $S$ phase significantly decreased in Hep-2/v cells compared to Hep-2 cells. This may be because VCR is able to bind to tubulin dimers, prevent the formation of spindles, and induce mitotic arrest in metaphase.

The development of MDR is a multifactorial process mediated by multiple mechanisms (3-9). Increased drug efflux resulting from the up-regulated expression of 

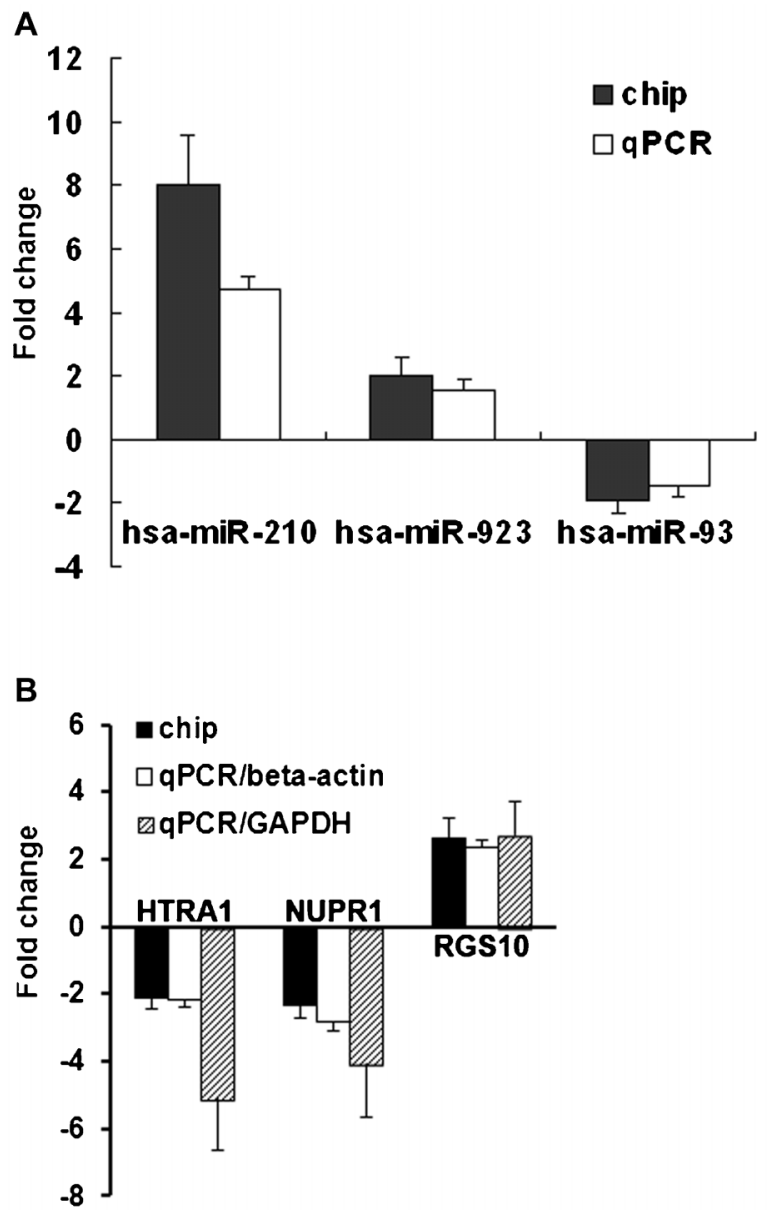

Figure 4. Real-time reverse transcriptase-polymerase chain reaction (RT-PCR) validating that the expressions of has-miR210 , has-miR-923, and regulator of G-protein signaling 10 (RGS10) are up-regulated and those of has-miR-93, hightemperature requirement protein A1 (HTRA1), and nuclear protein 1 (NUPR1) are down-regulated in Hep-2/v cells compared to Hep-2 cells. The relative levels of has-miR-210, has-miR-923, and has-miR-93 $(A)$, as well as HTRA1, NUPR1, and RGS10 transcripts $(B)$ in Hep-2 and Hep-2/v cells were determined by real-time RT-PCR. The relative expression levels of each microRNA were determined using U6 smalll nuclear RNA as an internal control, whereas those of each mRNA were determined using both beta-actin (q-PCR/beta-actin) and glyceraldehyde-3phosphate dehydrogenase ( $\mathrm{q}-\mathrm{PCR} / \mathrm{GAPDH}$ ) as endogenous controls. The fold-changes were calculated by dividing the expression level of each gene of interest relative to an endogenous control in Hep-2/v cells by that in Hep-2/v cells.

efflux drug transporters is a well-established cause of MDR (5). In this study, rhodamine 123 retention was significantly reduced and the expression of both MDR1 mRNA and protein was significantly up-regulated in Hep$2 / v$ cells, suggesting that drug efflux mediated by MDR1 overexpression is an important mechanism that allows Hep-2/v cells to acquire MDR.
A strong association between miRNAs and the acquisition of MDR by tumor cells has been established in previous studies $(15-20,26)$. In the present study, seven miRNAs were differentially expressed when Hep-2 cells were compared to Hep-2/v cells (Table 1). Of these identified miRNAs, has-miR-210 has been implicated in tumor hypoxia and drug resistance in head and neck squamous cell carcinoma $(27,28)$, has-miR-923 has been found to be up-regulated in taxol-resistant cancer cells (29), and has-miR-93 has been shown to be downregulated in adriamycin-resistant breast cancer MCF-7 cells (30). These findings, together with our observation, highlight the possible role of these miRNAs in MDR in LC.

MiRNAs exert their functions by modulating their target genes $(10,11)$. For this reason, we performed a cDNA microarray analysis to identify mRNAs that are differentially expressed between Hep-2 and Hep-2/v cells in order to predict putative target genes for has-miR-210, has-miR923, and has-miR-93. Of the 605 differentially expressed genes identified, three (HTRA1, NUPR1, and RGS10) were putative target genes for these miRNAs. Interestingly, Chien et al. (31) demonstrated that up-regulation of HTRA1 expression attenuates DDP- and paclitaxel-induced cytotoxicity, and, conversely, the forced expression of HTRA1 enhances DDP- and paclitaxel-induced cytotoxicity. This result is consistent with our finding that HTRA1 expression was down-regulated in multidrug-resistant Hep-2/v cells, suggesting that HTRA1 down-regulation that is mediated by has-miR-210 may be involved in the acquisition of MDR by Hep-2/v cells.

NUPR1 was down-regulated in Hep-2/v cells and may be regulated by both has-miR-210 and has-miR-923. Some studies have demonstrated that NUPR1 can protect some cancer cells from apoptosis and confer resistance to some chemotherapeutic drugs $(32,33)$. In contrast, other studies have shown an inverse relationship between NUPR1 overexpression and apoptosis in different types of cancer $(34,35)$. These findings suggest a dual role of NUPR1 in regulating apoptosis and chemoresistance of cancer cells. RGS10 was up-regulated in Hep-2/v cells and may represent a novel MDR-associated protein, since, to date, no reports have shown a link between RGS10 expression and the chemoresistance of cancer cells.

In conclusion, we have developed a multidrugresistant human LC subline designated Hep-2/v by exposure of Hep-2 cells to stepwise increasing concentrations of VCR. Using this cell model, we performed microarray assays to compare the miRNA and mRNA expression profiles of Hep-2 and Hep-2/v cells and identified seven differentially expressed miRNAs and 605 differentially expressed mRNAs. Additionally, we explored the association between these differentially expressed miRNAs and mRNAs. These findings may open a new avenue for the clarification of mechanisms responsible for MDR in LC. 
Table 2. Representative mRNAs that are differentially expressed between Hep-2 and Hep-2/v cells.

\begin{tabular}{lcccc}
\hline Genbank accession No. & Full gene name & Gene symbol & Fold-change & q-value \\
\hline Up-regulated & & & \\
NM_016109 & Angiopoietin-related protein 4 & ANGPTL4 & 15.04 & 0.00 \\
NM_002228 & Transcription factor AP-1 & JUN & 7.92 & 0.00 \\
NM_000927 & Multidrug resistance protein 1 & ABCB1 & 5.87 & 0.14 \\
NM_139317 & Baculoviral IAP repeat-containing protein 7 & BIRC7 & 4.29 & 0.14 \\
NM_002925 & Regulator of G-protein signaling 10 & RGS10 & 2.61 & 0.14 \\
NM_006545 & Tumor suppressor candidate 4 & TUSC4 & 2.57 & 0.00 \\
Down-regulated & & & 0.48 & 0.50 \\
NM_006754 & Synaptophysin-like protein 1 & SYPL1 & 0.47 & 0.62 \\
NM_002775 & High-temperature requirement protein A1 & HTRA1 & 0.46 & 0.24 \\
NM_133377 & Cell cycle checkpoint protein 1 & RAD1 & 0.43 & 0.62 \\
NM_012385 & Nuclear protein 1 & NUPR1 & 0.18 & 0.00 \\
NM_001259 & Cell division protein kinase 6 & CDK6 & 0.06 & 0.00 \\
NM_021073 & Bone morphogenetic protein 5 & BMP5 & & \\
\hline
\end{tabular}

\section{Supplementary Material}

Click here to view Figures [pdf]

Click here to view Table [xls]

\section{References}

1. Carew JF, Shah JP. Advances in multimodality therapy for laryngeal cancer. CA Cancer J Clin 1998; 48: 211-228, doi: 10.3322/canjclin.48.4.211.

2. Bier H. Chemotherapeutic drug resistance in the management of head and neck cancer. Eur Arch Otorhinolaryngol 1993; 250: 200-208, doi: 10.1007/BF00171524.

3. Stavrovskaya AA. Cellular mechanisms of multidrug resistance of tumor cells. Biochemistry 2000; 65: 95-106.

4. Laberge RM, Karwatsky J, Lincoln MC, Leimanis ML, Georges E. Modulation of GSH levels in ABCC1 expressing tumor cells triggers apoptosis through oxidative stress. Biochem Pharmacol 2007; 73: 1727-1737, doi: 10.1016/ j.bcp.2007.02.005.

5. Higgins CF. Multiple molecular mechanisms for multidrug resistance transporters. Nature 2007; 446: 749-757, doi: 10.1038/nature05630.

6. Estrela JM, Ortega A, Obrador E. Glutathione in cancer biology and therapy. Crit Rev Clin Lab Sci 2006; 43: 143181, doi: $10.1080 / 10408360500523878$.

7. Kang MR, Chung IK. Down-regulation of DNA topoisomerase Ilalpha in human colorectal carcinoma cells resistant to a protoberberine alkaloid, berberrubine. Mol Pharmacol 2002; 61: 879-884, doi: 10.1124/mol.61.4.879.

8. Keshelava N, Zuo JJ, Chen P, Waidyaratne SN, Luna MC, Gomer CJ, et al. Loss of p53 function confers high-level multidrug resistance in neuroblastoma cell lines. Cancer Res 2001; 61: 6185-6193.

9. Brozovic A, Majhen D, Roje V, Mikac N, Jakopec S, Fritz G, et al. Alpha(v)beta(3) integrin-mediated drug resistance in human laryngeal carcinoma cells is caused by glutathionedependent elimination of drug-induced reactive oxidative species. Mol Pharmacol 2008; 74: 298-306, doi: 10.1124/

\section{Acknowledgments}

We thank Medjaden Bioscience, Limited, for assisting in the preparation of this manuscript. mol.107.043836.

10. Bartel DP. MicroRNAs: genomics, biogenesis, mechanism, and function. Cell 2004; 116: 281-297, doi: 10.1016/S00928674(04)00045-5.

11. Ambros V. The functions of animal microRNAs. Nature 2004; 431: 350-355, doi: 10.1038/nature02871.

12. Calin GA, Croce CM. MicroRNA-cancer connection: the beginning of a new tale. Cancer Res 2006; 66: 7390-7394, doi: 10.1158/0008-5472.CAN-06-0800.

13. Kent OA, Mendell JT. A small piece in the cancer puzzle: microRNAs as tumor suppressors and oncogenes. Oncogene 2006; 25: 6188-6196, doi: 10.1038/sj.onc. 1209913

14. Lu J, Getz G, Miska EA, Alvarez-Saavedra E, Lamb J, Peck D, et al. MicroRNA expression profiles classify human cancers. Nature 2005; 435: 834-838, doi: 10.1038/nature03702.

15. Climent J, Dimitrow P, Fridlyand J, Palacios J, Siebert R, Albertson DG, et al. Deletion of chromosome 11q predicts response to anthracycline-based chemotherapy in early breast cancer. Cancer Res 2007; 67: 818-826, doi: 10.1158/ 0008-5472.CAN-06-3307.

16. Kovalchuk O, Filkowski J, Meservy J, Inytskyy Y, Tryndyak VP, Chekhun VF, et al. Involvement of microRNA-451 in resistance of the MCF-7 breast cancer cells to chemotherapeutic drug doxorubicin. Mol Cancer Ther 2008; 7: 21522159, doi: 10.1158/1535-7163.MCT-08-0021.

17. Xia L, Zhang D, Du R, Pan Y, Zhao L, Sun S, et al. miR-15b and miR-16 modulate multidrug resistance by targeting BCL2 in human gastric cancer cells. Int J Cancer 2008; 123: 372-379, doi: 10.1002/ijc.23501.

18. Yang H, Kong W, He L, Zhao JJ, O'Donnell JD, Wang J, et al. MicroRNA expression profiling in human ovarian 
cancer: miR-214 induces cell survival and cisplatin resistance by targeting PTEN. Cancer Res 2008; 68: 425-433, doi: 10.1158/0008-5472.CAN-07-2488.

19. Fujita $Y$, Kojima K, Ohhashi R, Hamada N, Nozawa $Y$, Kitamoto A, et al. MiR-148a attenuates paclitaxel resistance of hormone-refractory, drug-resistant prostate cancer PC3 cells by regulating MSK1 expression. J Biol Chem 2010; 285: 19076-19084, doi: 10.1074/jbc.M109.079525.

20. Zhu H, Wu H, Liu X, Evans BR, Medina DJ, Liu CG, et al. Role of microRNA miR-27a and miR-451 in the regulation of MDR1/P-glycoprotein expression in human cancer cells. Biochem Pharmacol 2008; 76: 582-588, doi: 10.1016/ j.bcp.2008.06.007.

21. Yang YX, Xiao ZQ, Chen ZC, Zhang GY, Yi H, Zhang PF, et al. Proteome analysis of multidrug resistance in vincristineresistant human gastric cancer cell line SGC7901/VCR. Proteomics 2006; 6: 2009-2021, doi: 10.1002/pmic.200402031.

22. Hong L, Wang J, Han Y, Zhao Y, Gao J, Wang J, et al. Reversal of multidrug resistance of vincristine-resistant gastric adenocarcinoma cells through up-regulation of DARPP-32. Cell Biol Int 2007; 31: 1010-1015, doi: 10.1016/ j.cellbi.2007.03.020.

23. Du B, Zhang $\mathrm{Y}$, Tang $\mathrm{Y}$, Wang $\mathrm{P}$. Minocycline attenuates ototoxicity and enhances antitumor activity of cisplatin treatment in vitro. Otolaryngol Head Neck Surg 2011; 144: 719-725, doi: 10.1177/0194599810395090.

24. Hong L, Zhao Y, Wang J, Han Y, Guo W, Jin H, et al. Reversal of multidrug resistance of adriamycin-resistant gastric adenocarcinoma cells through the up-regulation of DARPP-32. Dig Dis Sci 2008; 53: 101-107, doi: 10.1007/ s10620-007-9829-x.

25. Isaacs JT. Relationship between tumor size and curability of prostatic cancer by combined chemo-hormonal therapy in rats. Cancer Res 1989; 49: 6290-6294.

26. Blower PE, Chung JH, Verducci JS, Lin S, Park JK, Dai Z, et al. MicroRNAs modulate the chemosensitivity of tumor cells. Mol Cancer Ther 2008; 7: 1-9, doi: 10.1158/1535-7163.
MCT-07-0573.

27. Mathew LK, Simon MC. Mir-210: a sensor for hypoxic stress during tumorigenesis. Mol Cell 2009; 35: 737-738, doi: 10.1016/j.molcel.2009.09.008.

28. Gee HE, Camps C, Buffa FM, Patiar S, Winter SC, Betts G, et al. Hsa-mir-210 is a marker of tumor hypoxia and a prognostic factor in head and neck cancer. Cancer 2010; 116: 2148-2158.

29. Zhou M, Liu Z, Zhao Y, Ding Y, Liu H, Xi Y, et al. MicroRNA$125 \mathrm{~b}$ confers the resistance of breast cancer cells to paclitaxel through suppression of pro-apoptotic $\mathrm{Bcl}-2$ antagonist killer 1 (Bak1) expression. J Biol Chem 2010; 285: 21496-21507, doi: 10.1074/jbc.M109.083337.

30. Cui XY, Guo YJ, Yao HR. [Analysis of microRNA in drugresistant breast cancer cell line MCF-7/ADR]. Nan Fang Yi Ke Da Xue Xue Bao 2008; 28: 1813-1815.

31. Chien J, Aletti G, Baldi A, Catalano V, Muretto P, Keeney $\mathrm{GL}$, et al. Serine protease HtrA1 modulates chemotherapyinduced cytotoxicity. J Clin Invest 2006; 116: 1994-2004, doi: $10.1172 / \mathrm{JCl} 27698$.

32. Clark DW, Mitra A, Fillmore RA, Jiang WG, Samant RS, Fodstad O, et al. NUPR1 interacts with p53, transcriptionally regulates p21 and rescues breast epithelial cells from doxorubicin-induced genotoxic stress. Curr Cancer Drug Targets 2008; 8: 421-430, doi: 10.2174/156800908785133196.

33. Chowdhury UR, Samant RS, Fodstad O, Shevde LA. Emerging role of nuclear protein 1 (NUPR1) in cancer biology. Cancer Metastasis Rev 2009; 28: 225-232, doi: 10.1007/s10555-009-9183-X.

34. Ito $\mathrm{Y}$, Yoshida $\mathrm{H}$, Motoo $\mathrm{Y}$, lovanna JL, Nakamura $\mathrm{Y}$, Kakudo K, et al. Expression of p8 protein in breast carcinoma: an inverse relationship with apoptosis. Anticancer Res 2005; 25: 833-837.

35. Su SB, Motoo Y, lovanna JL, Berthezene P, Xie MJ, Mouri $\mathrm{H}$, et al. Overexpression of $\mathrm{p} 8$ is inversely correlated with apoptosis in pancreatic cancer. Clin Cancer Res 2001; 7 : 1320-1324. 